\title{
Shared annotations: the social side of exam preparation
}

\author{
Andrea Mazzei ${ }^{1}$, Jan Blom ${ }^{1}$, Louis Gomez ${ }^{2}$, and Pierre Dillenbourg ${ }^{1}$ \\ 1 CHILI, Ecole Polytechnique Fédérale de Lausanne, 1015 Lausanne, Switzerland \\ andrea.mazzei, jan.blom, pierre.dillenbourgdepfl.ch \\ ${ }^{2}$ Learning Sciences, University of California, Los Angeles CA 90095-1521, USA \\ lmgomez@ucla.edu
}

\begin{abstract}
In this paper we show how the deployment of a lightweight note sharing system can restore the antique social vocation of annotations in the classroom. The system was designed for the classroom context and evaluated through a longitudinal study lasting for an academic semester and involving 20 participants, enrolled in a Master-level course in computer science. Three key findings emerged. First, the tool spontaneously became an integral part of the classroom learning practices. Students took and shared annotations during the lectures and used them as complementary preparation material for the exam. Second, a correlation was observed between the annotation browsing time and the final exam grade. Third, a social bias emerged in favor of accessing one's own and friends' annotations. Based on the results, we discuss potential design implications for the system.
\end{abstract}

Keywords: Shared Annotations, Enhanced Learning, Social Bias

\section{Introduction and Motivation}

The social vocation of annotations slipped away after the advent of the printing press. Originally the manuscripts were annotated only by relevant academic authors. The produced glosses were shared to facilitate the scholarly need for elucidation and reinterpretation of the obscure passages and terminology of the texts. Nowadays students take notes individually, and engage occasionally in the spontaneous practice of note sharing through informal meetings, email or other forms of electronic communications. Communication features explicitly aimed at facilitating such note sharing practices could have benefits on the individual learning, group cohesion and team building. In the last semi-centennial we have assisted to the emerging evidence that note taking, in its different forms and fashions, serves both as an encoding and an external storage function [30]. Taking notes can trigger a deeper processing of information [5] and therefore increase learning and recall performance [12]. It has been also observed that annotations, in the form of visual arguments, can encode textual information in spatial forms that require a lower understanding effort [31] and help users in establishing relationships between the content and their mental constructions [17]. These theoretical foundations and empirical observations gave us the motivation in developing annOot, a system for sharing and browsing annotated instructional materials. This tool was embedded within the information system of the Swiss Federal Institute of Technology, Lausanne, and used by 20 students to reinforce the content associated with a Master's level computer science 
course. During the course, each student was provided with a tablet and a digital pen for reading and annotating the course material. The system collected both the produced annotations and the browsing history data for the entire semester. Through the analysis of these data we discuss the spontaneous adoption of annOot into the classroom learning practices. Then we point out salient navigation patterns and speculate on the social impact that shared annotations have within a community of learners.

\section{Related Literature}

\subsection{The vanishing gap between paper and digital annotations}

The mid 90s are known for the explosive digitalization of the publishing industry. At that time some of the widely used commercial authoring tools came to include features for in-context digital annotating. However, many authors observed and questioned the surprisingly poor adoption of web-based forms for annotating in the educational and work contexts [10]. O'Hara and Sellen [24] formalized the advantages that paper can offer to the annotation process, compared to computer-based interfaces, which were claimed to be cumbersome and to present an obstacle for the deep reading process. They also discussed major design implications for digital reading devices based on a seamless integration of annotating and reading. In a similar fashion, many of the commercial computer-interfaced authoring tools for annotation were taken under examination in order to define a set of design recommendations for note-taking applications [25]. Later Wolfe proposed a framework for discussing annotation technologies [33]. She spanned and classified many technologies for producing external textual attachments to any digital document. She concluded by shifting the focus of the discussion towards the pen-tablet XLibris [28], which was, at the time, a novel annotation tool able to bring some of the paper affordances and digital system capabilities into the reading process. Its ergonomic design succeeded in mimicking some of the physical properties of paper annotations. A usability test reported negligible differences on the reading habits using XLibris compared to the pen-and-paper [19]. More commercial solutions are available nowadays, some of those that were experimented in the classroom context, are reported in Section 2.2.

A different approach was initiated by Wellner with the DigitalDesk [32]. He came up with the idea of using paper as interface over digital resources. A camera-projector system was used to identify and track both the paper and the user inputs and to augment them with a visual feedback. Such an interaction paradigm blurs the line between paper and digital maintaining all the physical affordances of paper and including many of the valuable strengths of digital forms and tools. Optical acquisition and character recognition constituted a viable technological base for paper-based interfaces. They allowed the integration of storing, retrieving, and manipulating capabilities into paper documents [27], and linking of on-line daily planners with templated paper agendas [13]. More recently Bonnard et al. [3] showed how paper interfaces can be easily adopted into the traditional classroom practices. The miniaturization of commercial cameras, that allowed the integration of the optical acquisition within the writing implement giving birth to the era of digital pen. A commercial implementation of such a device is Anoto, a digital pen that incorporates a small camera on the tip. These devices allowed the 
emergence of many frameworks for taking digital annotations on paper texts [11] and for sharing them with teachers and students [18].

\subsection{Research on social annotations}

Digital annotation tools introduced a social component in the annotation practices. The CSCL community took the opportunity to incorporate such social forms of annotation to facilitate the teaching-learning processes. For instance, Wellner explored remote collaborative writing with Digital Desk [32]. Davis and Huttenlocher [7] reported a longitudinal study concerning CoNote; a web-based system that enabled users to access on-line course discussions via shared annotations. The system was initially deployed in the course of computer science and then became integral part of the teaching tools instantiated for that course. The authors reported anecdotal evidence on the usefulness of the tool in learning the course material and how easily the annotations were integrated within the course material. Crossen et al. [6] experimented a reader awareness system, using the XLibris tablet [28], in a high school classroom, reporting emerging needs from usability perspectives both on students and instructor sides. Another tablet-based device for note taking which was successfully experimented in classroom lectures was NotePal [8]. Marshall and Brush [20] investigated what type of annotating practices were more likely to be shared and how the transition from personal to public annotations should be mediated by mobile technology. Miura et al. [22] experimented the system AirTransNotes for augmenting in-classrooms activities using shared notes. A successful use of tablet for cooperative note-taking was also reported by Kam et al. [15]. Tabletsmediated communication was also successfully experimented in the classroom to gather students annotations as feedback of the teaching activity $[1,2]$.

\section{3 annOot}

As reported in the literature review, both paper- and tablet-based applications can constitute valid settings for a seamless integration between in-classroom note-taking and the teaching activities. However it seems that More Schools Embrace the iPad as a Learning Tool [14]. For our technological setting we decided to opt for a tablet-and-pen ${ }^{3}$ based solution because it provides ergonomic features for taking notes in symbiosis with the learning space and compatible with situated learning practices. Also it brings all the browsing, searching and storing features of mobile computers. This setting was coupled with the web-based system annOot, that we specifically designed, developed and deployed in the longitudinal study, reported in Section 5. annOot is a system providing browsing capabilities of the annotated course material. The system offers learners with an exhaustive and enhanced navigation of each other's digital notes. The users of annOot can take notes directly on the tablet, using commercial applications, during the lecture (as exemplified in Figure 1). Then they can revise the annotations and later decide to upload them on the annOot server using proprietary means of sharing. After every lecture each user can browse all the shared annotated material by using the web-based graphical user interface of the tool (see figure 2).

\footnotetext{
${ }^{3}$ Second generation iPad coupled with a Just Mobile AluPen
} 

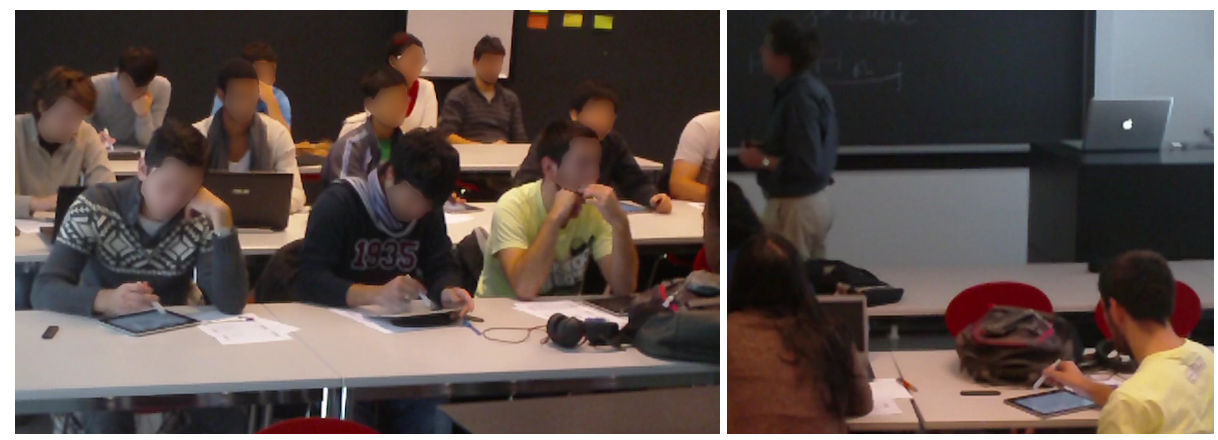

Fig. 1. Note-taking during lectures using tablets.

\subsection{Server Architecture}

The server side embodies a database containing each single page or slide of the digital instructional material of the course. It can collect all the shared annotated documents via HTTP receiver or by polling a dedicated file sharing system. When the user decides to share an annotated document the corresponding document images are submitted to the server. The server receives and processes them, using a computer vision procedure, in order to separate the annotations from the original document. More technically each shared page/slide is first recognized using locally likely arrangement hashing [23]. Second, it is rectified based on the estimated planar pose [9]. Finally a subtraction procedure, resistant to moderate misalignment, is applied to the rectified annotated document image in order to extract the layer containing the annotations.

\subsection{Graphical User Interface}

A student can access a specific annotated slide by using annOot. He or she has to go through the following five steps, highlighted in the five labeled areas in Figure 2.

1. Enter the url annoot.epfl.ch. The address shows that the system belongs to the realm of EPFL web information systems. We decided to deploy annOot as EPFL web service in order to exhibit credentials trustful for the users.

2. Select the visualization modality. The individual modality allows users to visualize all the annotated material for a specific annotator. The group modality allows users to visualize all the annotations for a specific page. The Moodle button allows users to go to the page of the course containing all the annotation-free course material.

3. Choose the lecture date. Each button corresponds to a given lecture of the course.

4. Choose the slide number. Once clicked on a chosen lecture date, the user can display a specific annotated slide by clicking on the slide menu located in the bottom of the page. The menu looks like a histogram. Each bar of the histogram corresponds to a slide of the lecture material. The height of each bar quantifies the amount of annotations produced for that slide. Left and right arrow keys were also included as navigation hot keys for scrolling the slide show. 


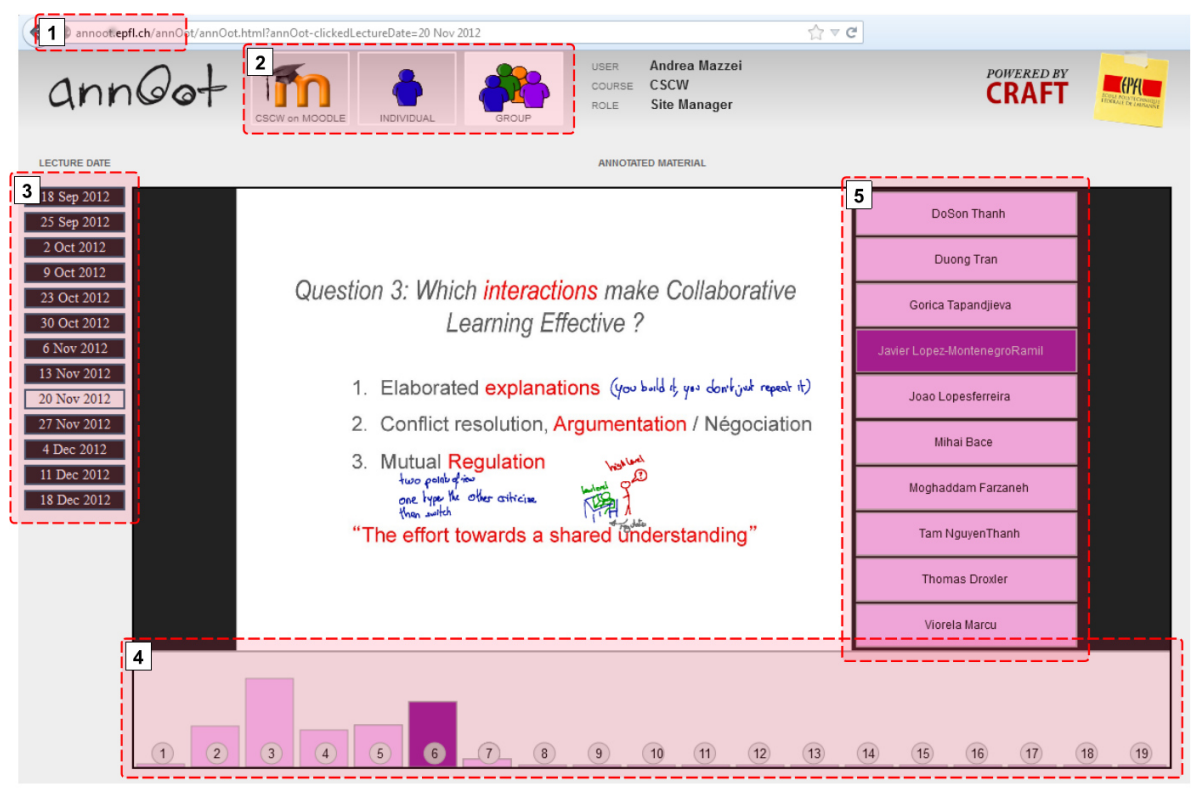

Fig. 2. annOot graphical user interface.

5. Select the annotator name. The user can display the annotations of a student by clicking on his or her name in the menu displayed on the right side of the page. Through this panel a user can immediately recognize his or her own and friends' annotations. In Section 6.4 we discuss how this panel can convey a social bias affecting the user browsing behaviour.

To summarize, we see the following two modes of usage characterizing the system. First, annotations can be made to the lecture handouts when listening to the lecture. Second, the taught content can be reinforced at any given time by reading through the lecture slides and accompanying annotations. In the next two sections we state our research hypotheses concerning the usage and utility of the tool and we describe the study that we have conducted in order to assess them.

\section{Research Questions and Hypotheses}

Two main user practices concern annOot: note taking in the classroom and browsing shared annotated material after the lecture. Our very first question was centered on a potential relationship between these two user behaviours.

$\mathrm{Q}_{\mathrm{A}}$. How does note-taking affect the browsing behaviour of annotated slides?

- $H_{1}$. The number of shared annotations, taken during a lecture, has a positive relationship with the time spent browsing all the annotations taken for that lecture. 
Second, we reconsidered the theoretical and empirical foundations sustaining that, taking notes [30,5, 12], and reading in presence of external representations [31, 17], enhance learning and retention. Therefore we wondered whether using annOot while taking and sharing notes and browsing shared notes would be beneficial for students comprehension.

$\mathrm{Q}_{\mathrm{B}}$. How does a frequent usage of the annOot system affect the student learning performance?

- $\mathrm{H}_{2}$. The number of notes taken during a lecture, being aware of sharing them later, is positively correlated with the final exam grade.

- $H_{3}$. The time spent browsing the shared annotations as complementary exam preparation practice is positively correlated with the final exam grade.

Third, considering the increasing evidence that information propagation in social networks is subject to the homophily principle [21], we investigated whether the classroom social boundaries influence the browsing behaviour of the shared annotated material.

$Q_{C}$. How does the social influence affect the navigational flow of shared annotated material? We expect that a friendship bias might influence the way a user opens a new slide by first clicking on the name of the annotator to visualize the annotations. This could be investigated by the following formal hypotheses:

- $H_{4}$. The empirical probability of first clicking on an annotation produced by the annotator him/herself is higher than the a priori theoretical probability.

- $H_{5}$. The empirical probability of first clicking on an annotation produced by a friend of the annotator is higher than the a priori theoretical probability.

- $H_{6}$. The empirical probability of first clicking on an annotation produced by a non-friend of the annotator is lower than the a priori theoretical probability.

In addition, we expected a friendship bias also on the browsing time of the annotations:

- $H_{7}$. The time spent browsing an annotation produced by a friend is higher than the time spent browsing an annotation produced by a non-friend classmate.

- $H_{8}$. The time spent browsing one's own annotation is lower than the time spent navigating an annotation produced by anyone else.

\section{Study Design}

A longitudinal study, starting at the beginning of October 2012 and ending at the end of January 2013, was conducted in order to address the hypotheses postulated in section 4 .

\subsection{Participants and Instructional Material}

The group of Master students, at the Swiss Federal Institute of Technology Lausanne, that enrolled in the course Computer Supported Cooperative Work in the fall semester 2012 was invited to participate in the study: 20 students accepted to participate, 4 declined their interest in the experiment, 1 dropped the course. Among the participants there were 5 females and 15 males. Their age ranged between 21 and 28 years old $(\mathrm{M}=23.6, \mathrm{SD}$ $=1.18$ ). The course material is constituted by 13 sets of slides, counting a total of 386 slides. Complementary resources referring to books and media contents were indicated on the Moodle page of the website. 


\subsection{Procedure}

The study was organized during the fall semester 2012. At the beginning, each participant was given a tablet and a digital pen and was instructed on how to download the course material onto the tablet and how to use it to take notes. A commercial free application for note taking was suggested, however all participants were free to choose their preferred one. Finally they were explained the necessary procedural steps for sharing notes and introduced to the main features of annOot browsing capabilities. All participants could keep the tablet as personal device for the entire semester; however they were asked to bring it to the classroom for the lectures. At the end of the semester each student was evaluated via formal oral examination. The examination committee was composed of two teachers of the course and an external expert in the field. The examiners elaborated 24 open-ended comprehension questions covering the content of the course material. During this examination instance, each student randomly chose two questions. The three examiners had to reach a consensus on the final grade, considering the appropriateness and completeness of the student's answers. All grades were expressed in the numeric range between 1.0 and 6.0, with a minimum passing threshold of 4.0.

\subsection{Data}

The collected data can be divided among three classes: (1) system usage, (2) social network; (3) mapping between lecture handout content and exam questions.

System Usage Two types of data were recorded from the participants. First, all the annotations were collected from the students for all the course lectures, and stored in annOot database. Second a user tracking system was developed in order to collect data from the usage of annOot navigation tool. For each action performed on annOot, the following information were recorded: the time-stamp, the user identifier, the visualization mode, the lecture identifier, the browsed slide and selected annotator's name.

In order to shed light on the user browsing behavior of shared annotated slide an inferred variable was computed: the annotated slide browsing time $(A B T)$. This variable measures how much time the user spends browsing the shared annotated slides. The variable is calculated by subtracting two sequential timestamps referring to two different browsed annotation identifiers and the same user identifier. We performed a measurement check of the $A B T$ in order to filter out some noise from the data. For instance a user could forget to close the page of the browsed annotated slide creating artificially long navigation times. Therefore we first imposed a maximum threshold on the $A B T$ of 5 minutes. In addition, during some lab tests we observed that the user could be tempted, by some navigational properties of the graphic user interface, to rapidly browse the annotated material without paying attention to the content of the annotations. To verify this observation we magnified the histogram of the $A B T$ in a narrow range comprised between 0 and 5 seconds (see figure 3 ). The histogram revealed the existence of a first peak at $200 \mathrm{~ms}$ circa. This peak contains all the $A B T$ data entries that are shorter than the average reading fixation time of $250 \mathrm{~ms}$ [29]. Therefore we concluded that all these short $A B T$ were not representative of the meaning expressed by the variable and we filtered them out by imposing a minimum threshold of half a second. 


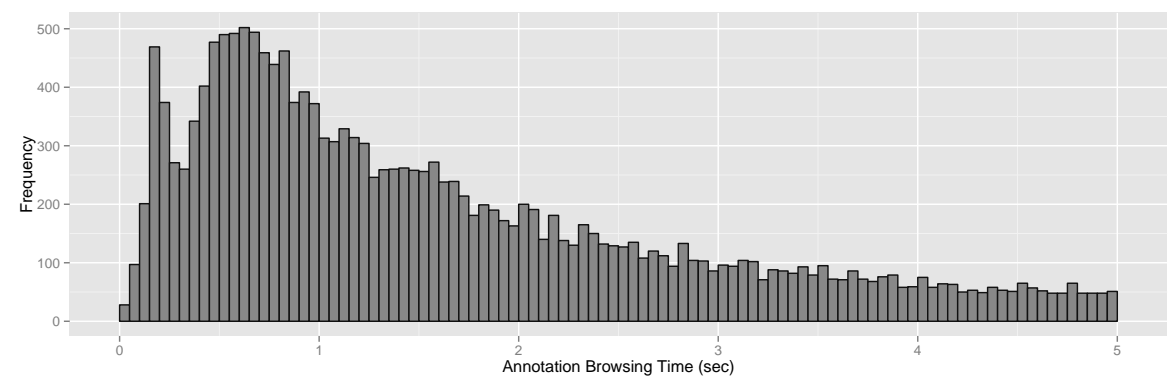

Fig. 3. Histogram of the $A B T$ in the range $0-5 \mathrm{sec}$.

Friendship Cognitive Social Structure As formally expressed in Section 4, a perceived friendship bias in the user browsing behavior was expected. To address this research hypothesis it was necessary to construct a data structure modeling the friendship ties in the classroom. The experimented release of annOot did not include any social networking capability. Therefore at the end of the course we decided to collect data on perceived friendship by using individual on-line surveys (we guaranteed anonymity conform to the data protection laws). Then we encoded the perceived friendship relationships, extracted from the on-line surveys, into cognitive social structures, by using means and modalities similar to the ones proposed by Krackhardt [16]. In this procedure we collected one friendship map for each respondent. This data structure allowed us to elaborate a categorical variable indicating the social provenience of a browsed annotation $(A S P)$. This variable indicates whether a browsed annotation was originally produced by ego (the user herself), or by friends or by non-friends. To obtain an overview of the friendship network of the classroom, we generated the friendship socio-gram of the locally aggregated structure [16]. The data structure is a simple graph $C=(S, F)$. Each node $S$ corresponds to a student. Each edge $F$ corresponds to a mutually perceived friendship; for instance the edge $e=\left(s_{i}, s_{j}\right)$ is the friendship expressed by the student $s_{i}$ with regards to the student $s_{j}$ and vice versa.

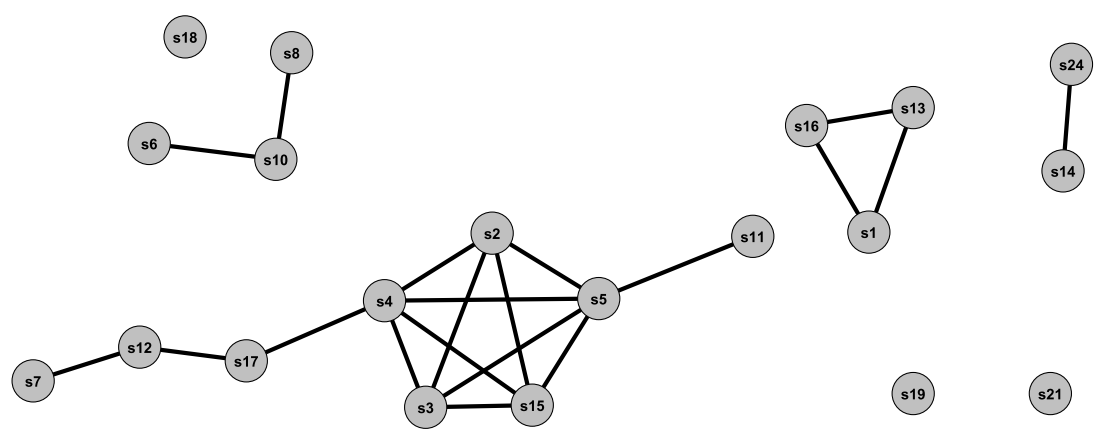

Fig. 4. Socio gram of the locally aggregated structure [16]. 
Mapping between handout content and exam questions. Chi [4] suggested a method for reducing the vulnerability of qualitative data analysis to subjective interpretations. The method applies to coding qualitative data. Inspired by this approach we elaborated a matching scheme between exam questions and instructional units. Each of the 24 exam questions was matched with the subset of lecture slides that should be read and understood in order to answer to the question itself. We modeled this question-slides dependency using a bipartite graph $G_{Q}=(Q, L, E) . Q=\left\{q_{1}, q_{2}, \ldots, q_{n}\right\}$ corresponds to the set of questions asked to the students during the examination session. $L=$ $\left\{l_{1}, l_{2}, \ldots, l_{k}\right\}$ corresponds to the set of slides released during the course. The bipartite graph was manually constructed by one of the authors, by linking the questions in $Q$ to the sets of lecture slides in $L$, such that if the understanding of the content of the set of slides $l_{i}$ is necessary to answer to the question $q_{j}$, the edge $e_{i, j}$ is added to the set $E$. This bipartite graph allowed us to recompute all experimental features only for the partitioned section of the instructional material relevant for answering the exam question.

\section{Results}

\subsection{Descriptive data}

We first report the quantitative data on the classroom participation in producing, sharing and browsing annotations. On average each student annotated $36.05(\mathrm{SD}=29.77)$ slides ( $10 \%$ of the total material). Considering the number of browsed annotated slides, each user accessed $1182(\mathrm{SD}=920)$ annotated slides. The user-based histograms showing the number of shared annotated slides in Figures 5(a) and the number of browsed slides in 5(b) suggest that almost all users participated in the usage of annOot. It is interesting to notice that those students who did not take or share any note still used annOot for browsing the slides annotated by the other students. In the time-based histogram in Figure 5(c) we show that the usage of the browsing tool was scarce during the semester and skyrocketed in the two weeks before the exam.

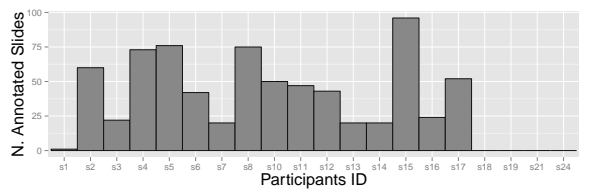

(a) Shared annotated slides per user.

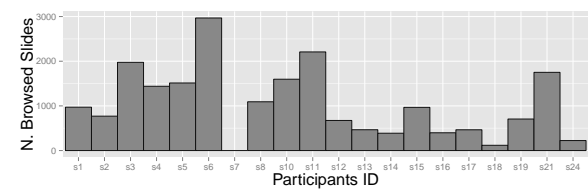

(b) Browsed slides per user.

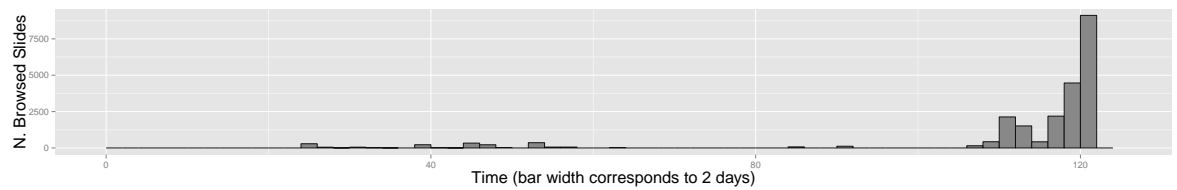

(c) Time-based histogram of the number browsed annotated slides.

Fig. 5. Descriptive statistics of students participation with annOot system. 


\subsection{The synergistic effect between note-taking and the note-browsing.}

We here address the research hypothesis $H_{1}$. Linear mixed effect regression was employed to measure the relationship between the number of annotated slides per lecture $\left(N u m A n n_{L E C}\right)$ and the browsing time of the annotated slides of the same lecture $\left(A B T_{L E C}\right)$. While the first activity is done in classroom, the second one is an out-ofclassroom learning experience. Given the nature of the test the user identifier was taken into account as grouping factor. The resulting linear model exhibits a significant intercept $(E s t .=688$, Std.E. $=268, t(57)=2.57, p=0.013)$ meaning that each user on average spends 11 minutes $(688 \mathrm{sec})$ in browsing the annotated slides of one specific lecture. Also the slope resulted do be positive and significant $(E s t .=54, S t d . E .=15$, $t(57)=3.53, p=0.0008$ ) indicating that the ABT is extended by roughly one additional minute $(54 \mathrm{sec})$ multiplied for the number of shared annotations during that lecture. We accept $H_{1}$.

\subsection{Student Examination Performance}

We here address the research hypotheses $\mathrm{H}_{2}$ and $\mathrm{H}_{3}$. For each student we took into account the two questions, $Q=\left\{q_{i}, q_{j}\right\}$, asked during the exam. We used the bipartite graph $G_{Q}=(Q, L, E)$ to select the subset of the instructional material $I\left\{i_{s}, i_{t}, \ldots, i_{u}\right\}$, which understanding was required to correctly answer to those two questions (as already explained in Section 5.3). Then for each student, we recalculated the number of annotated slides and the annotated slide browsing time relative to such partition of the instructional material, and we labeled the new variables respectively $N u m A n n_{R E L}$ and $A B T_{R E L}$.

A Kendall rank correlation test between $N u m A n n_{R E L}$ and the exam score was carried out ${ }^{4}$. The result is not significant $(\tau(18)=-0.10, p=0.53)$. We reject $H_{2}$. A second correlation test between $A B T_{R E L}$ and the exam score was conducted. For this second test we found a positive and significant correlation $(\tau(18)=0.32, p=$ 0.042). We accept $H_{3}$. This result encouraged us to elaborate a deeper investigation. We questioned whether the time spent browsing only the annotations produced by the best students of the classroom better correlates with the individual exam score. Therefore the student group was split in two sub-groups using the median of the exam score $\left(Q 2_{\text {ExamScore }}=4.95\right)$. For each student, the browsing annotation time was recomputed considering only the annotations produced by the best half of the classroom; the new variable is labeled as $A B T_{R E L, B E S T}$. A third correlation test between $A B T_{R E L, B E S T}$ and the exam score was conducted. The test revealed a strong positive and significant correlation $(\tau(18)=0.35, p=0.032)$. We can conclude that browsing the annotations produced by the best-in-classroom students is correlated with the individual exam grade.

\footnotetext{
${ }^{4}$ Kendall rank correlation test is a non-parametric test that does not assume any assumption related to the distributions and is robust to the presence of outliers. We opted for this correlation method because the Shapiro and Wilk normality test revealed that the variable exam score is not normally distributed ( $W=0.88, p=0.019$ ) and that the low sample size of the dataset $(N=20)$ could exhibit a higher exposure to the presence of outliers.
} 


\subsection{Friendship bias in browsing shared annotations}

When the user enters a new slide he or she can access to someone's annotations (produced and shared by ego, friends or non-friends, see Section 5.3) by clicking on her name. As stated in $H_{4}, H_{5}$ and $H_{6}$, we expect a social bias conveyed through the name list of annotators (see panel 5 in Figure 2). This bias can influence the user's decision of whose annotations should be accessed first. To verify this we compared the theoretical probabilities of randomly first clicking on one's own annotations (ego), on friends' annotations (friends) and on non-friends' annotations (non-friends) with the related measured empirical probabilities. For instance we computed the theoretical and empirical probabilities of first clicking on a friend's annotation on a specific slide as follows:

$$
P(\text { friends })=\frac{\mid \text { friends } \mid}{\mid \text { annotations } \mid} \quad F(\text { friends })=\frac{n_{\text {friends }}}{n_{\text {annotations }}}
$$

Considered a specific annotated slide, the theoretical probability of first clicking on a friend's annotation $P$ (friends) corresponds to the number of annotations produced by the user's friends $(\mid$ friends $\mid)$ divided by the total number of annotations produced by everybody for that slide (|annotations $\mid)$. The empirical probability of first clicking on a friend's annotation $F$ (friends) corresponds to the number of observed first clicked friends' annotations in that slide $\left(n_{\text {friends }}\right)$, divided by the total number of first clicked annotations $\left(n_{\text {annotations }}\right)$. Only the data entries in which the user could click on all the mentioned social categories were considered for this test. To measure any discrepancy between the empirical and theoretical probabilities, we employed linear mixed effect regression with probabilities as measured variable and with the categorical predictor indicating whether they are theoretical or empirical. Three models were built, one for each type of social category. A nested grouping factor accounting the slide identifier was introduced in the models. The empirical probability of first clicking on one's own annotations is higher $(F(E g o)-P(E g o)=0.11)$ than the correspondent theoretical probability $(t(433)=5.69, p<0.0001) . H_{4}$ is accepted. The empirical probability of first clicking on one's friends annotations is not significantly different $(F$ (friends) $P($ friends $)=0.0049)$ than the correspondent theoretical probability $(t(433)=0.23$, $p=0.81) . H_{5}$ is rejected. The empirical probability of first clicking on non-friends' annotations is lower $(F($ Others $)-P($ Others $)=-0.12)$ than the correspondent theoretical probability $(t(433)=-5.43, p<0.0001))$. $H_{6}$ is accepted.

As last step of the analysis we tested the research hypotheses $H_{7}$ and $H_{8}$. We investigated whether the social provenience of an annotation can influence its browsing time. Therefore we constructed a linear mixed-effects model where the ASP categorical variable predicts the $A B T$ numerical variable. The user identifier was taken into account as a grouping factor. The results show that the user spends less time on her/his own annotated slides $\left(A B T_{\text {ego }}-A B T_{\text {friends }}=-2.4 \mathrm{sec}\right)$ than on friends' ones $(t(11884)=-2.34, p=0.019)$. The user spends less time on non-friends' annotated slides $\left(A B T_{\text {non-friends }}-A B T_{\text {friends }}=-1.7 \mathrm{sec}\right)$ than on friends' ones $(t(11884)=-2.71, p=0.0067)$. We accept $H_{7}$. However the time spent non-friends' annotated slides is not different $\left(A B T_{\text {non-friends }}-A B T_{\text {ego }}=0.82 \mathrm{sec}\right)$ than the time spent on one's owns annotated slides $(t(11884)=-0.88, p=0.37)$. We reject $H_{8}$. 


\section{Discussion and Conclusion}

From the quantitative observations on the usage of the tool, we can conclude that students used the tablet for note-taking fairly regularly during the arc of the semester. This strengthen existing experimental and observatory investigations on the ability of tablets at mimicking the physical properties of paper in digital annotations [33, 14]. We also observed that students participated into the note sharing process and in browsing the shared annotations. Therefore our tool implemented Marshall's idea of mobile technology able to mediate the transition From Personal to Shared Annotations [20]. In addition we observed that annOot spontaneously broke the barrier of the in-classroom learning activities, since it was used by the students as complementary resource for the exam preparation. We also measured a synergistic effect between sharing the notes taken during a lecture and the time spent browsing the slides of the same lecture. We gave two plausible interpretations to this phenomenon: 1) taking and sharing notes increases students' engagement and motivation in browsing the notes taken from other classmates. 2) highly engaged students tend to browse often the course material and consequently take and share more notes. We are not able to discriminate any of these 2 interpretations.

We did not measure any effect of taking notes in classroom, using a tablet, on the exam grade. This result is interesting if considered that a vast research literature showed the positive effects of self-note-taking on learning performance [30,5, 12]. Clearly the limitations of the study prevent us from taking any strong conclusion on this result. However one interpretation could be that there is still room for improvement for tablet-based note-taking technologies. One immediate solution, easily integrable with our technological architecture, would rely on the integration of paper-based interfaces in annOot system [32,27, 13]. For instance the students could take notes on printed handouts and decide to share them by taking photos of the annotated areas using any mobile device. The recognition system integrated in annOot [23], can already robustly deal arbitrary perspectives in the document image.

Further on we showed how the time spent browsing the shared annotated instructional material before the exam, is positively correlated with the final exam score. This result is in line with a vast literature focusing on the effects of external and multiple representations on leaner's recall performance [31,17]. We would like to complement this result with an additional interpretation: the awareness of other students' annotative productions can disclose the emergence of a learning disequilibrium due to socio-cognitive interactions [26]. For instance the awareness of friends' and non-friends' annotations, can induce the students to improve, adapt, or complement their annotations and annotating behaviours. One interesting feature that should also be taken into account is the instructors' feedback. This type of feedback could reveal which passages of the instructional material are not well understood by the students. As already experienced by Crossen et al. [6], such feedback could result in modifications in the teaching work flow. Using our system the teacher can also easily integrate relevant notes to the teaching material of the subsequent year, as suggested by David et al. [8]. In addition we refined this latter result by observing that the time spent browsing the slides annotated by the best students of the classroom better correlates with the exam grade. Such an expertise bias could drive prioritization criteria in the visualization of the annotations. 
As last result we observed how the social provenience of shared annotations can affect the user browsing behaviour. The user tends to first click more often on his or her own annotations and less often on non-friends' annotations. We also found that the user spends more time on friends annotations than his owns' or the others'. This result shows that the students' browsing of socially labeled notes is biased in favour of strong ties; this means that annOot system modulates the interaction between the student and the annotated material concordantly with the classroom social boundaries. This modest manifestation of the homophily principle in social network analysis [21] could be compensated, in the future, by using content-based recommendation systems.

We conclude that the discussed results will encourage the deployment of annOot system as fully accessible University micro-publishing platform based on both digital and paper note-taking. We believe that the system can support fruitful learning activities, complementary to traditional means of lecturing. An evolution of the system should integrate a social network of the students where each attended course generates a potential circle of friends. In addition it should integrate a recommender system of authored or annotated course material, based on contents and students' proficiency.

\section{Acknowledgements}

David Bréchet has our utmost gratitude for his insightful assistance pertaining to information technology. We also thank all the students who participated in the study. This work is funded by NCCR IM2 (Interactive Multimodal Information Management, IM2-IP2).

\section{References}

1. Anderson, R., Davis, P., Linnell, N., Prince, C., Razmo, V., Videon, F.: Classroom presenter: Enhancing interactive education with digital ink. Computer. 40, 56-6 (2007)

2. Berque, D.: An evaluation of a broad deployment of DyKnow software to support note taking and interaction using pen-based computers. Journal of Computing Sciences in Colleges. 21, 204-21 (2006)

3. Bonnard, Q., Verma, H., Kaplan, F., Dillenbourg, P.: Paper Interfaces for Learning Geometry. In: Proceedings of EC-TEL '12, pp. 37-50. Springer Berlin Heidelberg, Heidelberg (2012)

4. Chi, M. T. H.: Quantifying qualitative analyses of verbal data: A practical guide. Journal of the Learning Sciences. 6, 271-315 (1997)

5. Craik, F. I. M., Lockhart R. S.: Levels of processing: A framework for memory research. Journal of Verbal Learning and Verbal Behavior. 11, 671-68 (1972)

6. Crossen, A., Budzik, J., Warner, M., Birnbaum, L., Hammond, K. J.: XLibris: an automated library research assistant. In: Proceedings of IUI '00, pp. 49-52. ACM Press, New York (2001)

7. Davis, J. R., Huttenlocher, D. E.: Shared annotation for cooperative learning. In: Proceedings of CSCL '95, pp. 84-88. L. Erlbaum Associates Inc., Hillsdale (1995)

8. Davis, R.C., Landay, J.A., Chen, V., Huang, J., Lee, R.B., Li, F.C., Lin, J., Morrey, C.B., III, Schleimer, B., Price, M.N., Schilit, B. N.: NotePals: Lightweight note sharing by the group, for the group. In: Proceedings of the CHI '99, pp. 338 345. ACM Press, New York, (1999).

9. Fischler, M. A., Bolles, R. C.: Random sample consensus: a paradigm for model fitting with applications to image analysis and automated cartography. Comm. ACM. 6, 381-395 (1981) 
10. Fu, X., Ciszek, T., Marchionini, G., Solomon, P.: Annotating the Web: An exploratory study of Web users' needs for personal annotation tools. In: Proceedings of ASIST '05. (2005)

11. Guimbretire, F.: Paper augmented digital documents. In: Proceedings of UIST '03, pp. 51-60. ACM Press, New York (2003)

12. Hartley J., Davies I.: Note-taking: A critical review. Innovations in Education and Teaching International, 15, 207-224 (1978)

13. Heiner, J. M., Hudson, S. E., Tanaka, K.: Linking and messaging from real paper in the Paper PDA. In: Proceedings of UIST '99, pp. 179-186. ACM Press, New York (1999)

14. Hu, W.: More Schools Embrace the iPad as a Learning Tool. The New York Times (2011)

15. Kam, M., Wang, J., Iles, A., Tse, E., Chiu, J., Glaser, D., Tarshish, O., Canny, J.: Livenotes: a system for cooperative and augmented note-taking in lectures. In: Proceedings of CHI '05, pp. 531-540. ACM Press, New York (2005)

16. Krackhardt, D.: Cognitive social structures. Social Networks, 9, 109-134 (1987)

17. Larkin, J. H., Simon, H. A.: Why a diagram is (sometimes) worth ten thousand words. Cognitive science, 11, 65-100 (1987)

18. Liao, C., Guimbretire, F., Hinckley, K., Hollan, J.: Papiercraft: A gesture-based command system for interactive paper. ACM Transactions on Computer-Human Interaction, 14, 18:118:27 (2008)

19. Marshall, C. C., Price, M. N., Golovchinsky, G., Schilit, B. N.: Introducing a digital library reading appliance into a reading group. In: Proceedings of DL '99, pp. 77-84. ACM Press, New York (1999)

20. Marshall, C. C., Brush, A. J. B.: From personal to shared annotations. In: CHI '02 Extended Abstracts, pp. 812-813. ACM Press, New York (2002)

21. McPherson, M., Smith-Lovin, L., Cook, J. M.: Birds of a feather: Homophily in social networks. Annual review of sociology. 27, 415-444 (2001)

22. Miura, M., Kunifuji, S., Shizuki, B., Tanaka, J.: AirTransNote: augmented classrooms with digital pen devices and RFID tags. In: Proceedings of WMTE '05, pp. 56 - 58. IEEE Computer Society, Washington (2005)

23. Nakai, T., Kise, K., Iwamura, M.: Use of affine invariants in locally likely arrangement hashing for camera-based document image retrieval. In: Proceedings of DAS '06, pp. 541552. Springer Berlin Heidelberg, Heidelberg (2006)

24. O'Hara, K., Sellen, A.: A comparison of reading paper and on-line documents. In: Proceedings of CHI '97, pp. 335-342. ACM Press, New York (1997)

25. Ovsiannikov, I. A., Arbib, M. A., McNeill, T. H.: Annotation technology. International Journal of Human-Computer Studies, 50, 329-362 (1999)

26. Piaget, J.: The psychology of intelligence. Routledge, London (2004)

27. Rao, R., Card, K., Johnson, W., Klotz, L., Trigg, R.: Protofoil: storing and finding the information worker's paper documents in an electronic file cabinet. In: Proceedings of CHI' 94, pp. 180-185. ACM Press, New York (1994)

28. Schilit, B. N., Golovchinsky, G., Price, M. N.: Beyond paper: supporting active reading with free form digital ink annotations. In: Proceedings of CHI' 98, pp. 249-256. ACM Press, New York (1998)

29. Sereno S.C., Rayner K.: The when and where of reading in the brain. Brain and Cognition, 42, 78-81 (2000)

30. Di Vesta, F., Gray, G. S.: Listening and note taking. Journal of educational psychology, 63, 8-14 (1972)

31. Waller, R.: Understanding network diagrams. Los Angeles (1981)

32. Wellner, P.: Interacting with paper on the DigitalDesk. Communications of the ACM, 36, 87-96 (1993)

33. Wolfe, J.: Annotation technologies: A software and research review. Computers and Composition, 19, 471-497 (2002) 